\title{
Weak decays of magnetized charged pions in the symmetric gauge
}

\author{
M. Coppola $\odot,{ }^{1,2}$ D. Gomez Dumm $\odot,{ }^{3}$ S. Noguera $\odot,{ }^{4}$ and N. N. Scoccola ${ }^{1,2}$ \\ ${ }^{1}$ CONICET, Rivadavia 1917, 1033 Buenos Aires, Argentina \\ ${ }^{2}$ Physics Department, Comisión Nacional de Energía Atómica, \\ Av. Libertador 8250, 1429 Buenos Aires, Argentina \\ ${ }^{3}$ IFLP, CONICET-Departamento de Física, Fac. de Cs. Exactas, Universidad Nacional de La Plata, \\ C.C. 67, 1900 La Plata, Argentina \\ ${ }^{4}$ Departamento de Física Teórica and IFIC, Centro Mixto Universidad de Valencia-CSIC, \\ E-46100 Burjassot (Valencia), Spain
}

(Received 28 October 2019; accepted 14 January 2020; published 5 February 2020)

\begin{abstract}
We consider the decay $\pi^{-} \rightarrow l \bar{\nu}_{l}\left(l=e^{-}, \mu^{-}\right)$in the presence of an arbitrary large uniform magnetic field, using the symmetric gauge. The consequences of the axial symmetry of the problem and the issue of angular momentum conservation are discussed in detail. In particular, we analyze the projection of both the canonical and the mechanical total angular momenta along the direction of the magnetic field. It is found that while the former is conserved in the symmetric gauge, the latter is not conserved in both the symmetric and Landau gauges. We derive an expression for the integrated $\pi^{-} \rightarrow l \bar{\nu}_{l}$ width that coincides exactly with the one we previously found using the Landau gauge, providing an explicit test of the gauge independence of that result. Such an expression implies that for nonzero magnetic fields the decay width does not vanish in the limit in which the outgoing charged leptons can be considered as massless, i.e., it does not exhibit the helicity suppression found in the case of no external field.
\end{abstract}

DOI: 10.1103/PhysRevD.101.034003

\section{INTRODUCTION}

Recently, a significant interest has been devoted to the effect of intense magnetic fields on the properties of strongly interacting matter [1-3]. This is mostly motivated by the realization that strong magnetic fields might play an important role in the study of the early Universe [4], in the analysis of high energy noncentral heavy ion collisions [5], and in the physics of stellar objects like the magnetars [6]. It is well known that magnetic fields induce interesting phenomena, such as the enhancement of the QCD vacuum (the so-called "magnetic catalysis") [7] and the decrease of critical temperatures for chiral restoration and deconfinement QCD transitions [8]. More recently, it has also been shown that an external magnetic field can lead to a significant enhancement of the leptonic decay widths of charged pions $[9,10]$. It should be noticed that the hadronic decay rates in external magnetic fields are important for the stability and equilibrium of magnetars. Moreover, their dominant cooling mechanisms involve (inverse) $\beta$-decay, photo-meson interactions and pion decay [11]. Pions radiate energy via inverse Compton scattering until they decay, imprinting the

Published by the American Physical Society under the terms of the Creative Commons Attribution 4.0 International license. Further distribution of this work must maintain attribution to the author(s) and the published article's title, journal citation, and DOI. Funded by SCOAP . spectrum of the subsequently produced neutrinos [12]. Although several studies of weak decays of hadrons under strong electromagnetic fields have been reported in the literature [13-16], in most works the effects of the external fields on the relevant hadronic matrix elements have not been fully considered. In the case of charged pions, these effects have been recently analyzed in the context of chiral perturbation theory [17], effective chiral models [18-21] and lattice QCD (LQCD) calculations [9]. The most general form of the relevant hadronic matrix elements for the processes $\pi^{-} \rightarrow l \bar{\nu}_{l}\left(l=e^{-}, \mu^{-}\right)$has been obtained through a model-independent analysis in Ref. [22], where the effect of a magnetic field $\vec{B}$ on both pion and lepton wave functions is fully taken into account. In particular, it is found that the vector and axial vector pion-to-vacuum matrix elements can be parametrized in general through one and three hadronic form factors, respectively. Taking into account the expression for the $\pi^{-} \rightarrow l \bar{\nu}_{l}$ decay width in Ref. [22], quantitative predictions have been given in Ref. [10] for magnetic fields up to $e B \simeq 1 \mathrm{GeV}^{2}$. Those results, based on the estimations given in Ref. [21] for the hadronic form factors and the pion mass within an effective Nambu-Jona-Lasino model, show a strong enhancement of the total width with respect to its value for $B=0$. In addition, it is seen that the presence of the magnetic field affects dramatically the ratio between muonic and electronic decay rates [10]. This is related to the fact that the widths do not vanish in the limit of vanishing lepton masses, as happens to be the case for $B=0$. 
For $\pi^{-} \rightarrow l \bar{\nu}_{l}$ decays, the presence of an external magnetic field has also an important effect on the angular distribution of outgoing antineutrinos. In Ref. [9], on the basis of some assumptions related to angular momentum conservation and chirality, it is claimed that the momentum $\vec{k}$ of the antineutrino has to be parallel to the magnetic field (i.e., the perpendicular component $\vec{k}_{\perp}$ has to be zero). This is in contradiction with the analysis in Ref. [10], where it is found that $\vec{k}_{\perp}$ is in fact the dominant component of the momentum for magnetic fields larger than about $0.1 \mathrm{GeV}^{2}$. According to Ref. [22], conservation laws do not imply $\vec{k}_{\perp}=0$, therefore one should integrate over all possible values of the antineutrino momentum. At this point it should be noticed that in Ref. [22] the calculation of the general form of the $\pi^{-} \rightarrow$ $l \bar{\nu}_{l}$ decay width has been carried out using expressions for the charged pion and lepton wave functions in the Landau gauge. Although this is in fact the most widely chosen gauge to perform this type of calculations, it may be not the most convenient one when dealing with arguments of angular momentum conservation, such as those considered in Ref. [9]. As noticed in Ref. [23], the consequences of the axial symmetry of the problem, as well as the physical meaning of angular momenta, can be better understood if one works in the symmetric gauge. Having this in mind, the purpose of the present work is to rederive the expression for the $\pi^{-} \rightarrow l \bar{\nu}_{l}$ decay width including the most general hadronic matrix elements in the presence of a uniform magnetic field, now considering the symmetric gauge. It is shown that this procedure enables a detailed discussion of angular momentum conservation issues in decay processes of magnetized charged particles. In addition, our calculation is found to confirm the expected gauge independence of the general expression for the decay width.

This paper is organized as follows. In Sec. II we present the expressions of the different charged fields used in our work and discuss in detail angular momentum and chirality properties. In Sec. III we obtain explicit expressions for the $\pi^{ \pm}$leptonic weak decay amplitudes. We consider both the general case of an arbitrary magnetic field strength and the limit of strong fields. Then we obtain the expression for the decay width by summing and integrating over all possible final states. Our main conclusions are presented in Sec. IV. We also include Appendixes A-D to quote some technical details of our calculations.

\section{PRELIMINARIES: CHARGED PARTICLE FIELDS UNDER A UNIFORM MAGNETIC FIELD IN THE SYMMETRIC GAUGE}

Let us start by quoting the expressions for the different charged fields considered in our work, written in terms of particle creation and annihilation operators. We use the
Minkowski metric $g^{\mu \nu}=\operatorname{diag}(1,-1,-1,-1)$, while for a space-time coordinate four-vector $x^{\mu}$ we adopt the notation $x^{\mu}=(t, \vec{r})$, with $\vec{r}=\left(r_{x}, r_{y}, r_{z}\right)$. Assuming the presence of a uniform magnetic field $\vec{B}$, we orientate the spatial axes in such a way that $\vec{B}=B \hat{r}_{z}$, and consider the symmetric gauge, in which $\mathcal{A}^{\mu}=(0, \vec{A})$ with $\vec{A}=\vec{r} \times \vec{B} / 2=$ $\left(-B r_{y} / 2, B r_{x} / 2,0\right)$. The expressions for the charged particle fields can be obtained using the method described e.g., in Sec. 19 of Ref. [24]. For the reader's convenience we quote here these expressions explicitly, since they are not commonly given in the literature (in comparison to those corresponding to the Landau gauge). In the last subsection we discuss some issues related to the quantum numbers of particle states.

\section{A. Charged pion field}

The charged pion fields can be written as

$$
\begin{aligned}
\phi_{\pi^{\sigma}}^{s}(x)= & \phi_{\pi^{-\sigma}}^{s}(x)^{\dagger} \\
= & \sum_{\ell, l=0}^{\infty} \int \frac{d p_{z}}{(2 \pi)^{3} 2 E_{\pi^{\sigma}}} \\
& \times\left[a^{\sigma}(\breve{p}) W_{\bar{p}}^{s}(x)+a^{-\sigma}(\breve{p})^{\dagger} W_{\bar{p}}^{-s}(x)^{*}\right],
\end{aligned}
$$

where $Q_{\pi^{\sigma}}=\sigma|e|$ is the pion charge with $\sigma= \pm, s=$ $\operatorname{sign}\left(Q_{\pi^{\sigma}} B\right)$ and $B_{e}=\left|Q_{\pi^{\sigma}} B\right|=|e B|$. Note that if $B>0$ then $s=\sigma$. The pion energy is given by $E_{\pi^{\sigma}}=$ $\sqrt{m_{\pi^{\sigma}}^{2}+(2 \ell+1) B_{e}+p_{z}^{2}}$. We have also defined $\bar{p}=$ $\left(E_{\pi^{\sigma}}, \breve{p}\right)$ and $\breve{p}=\left(\ell, l, p_{z}\right)$, where $p_{z}$ is an arbitrary real number while $\ell$ and $l$ are non-negative integer numbers. The functions $W_{\bar{p}}^{s}(x)$ are solutions of the eigenvalue equation

$$
\mathcal{D}_{\mu} \mathcal{D}^{\mu} W_{\bar{p}}^{s}(x)=-\left[E_{\pi^{\sigma}}^{2}-(2 \ell+1) B_{e}-p_{z}^{2}\right] W_{\bar{p}}^{s}(x),
$$

where $\mathcal{D}^{\mu}=\partial^{\mu}+i s B_{e}\left(r_{x} \delta^{\mu 2}-r_{y} \delta^{\mu 1}\right) / 2$. Introducing polar coordinates $\rho, \phi$ in the plane perpendicular to the magnetic field, their explicit form is given by

$$
W_{\bar{p}}^{s}(x)=\sqrt{2 \pi} e^{-i\left(E_{\pi^{\sigma}} t-p_{z} r_{z}\right)} e^{-i s(\ell-\imath) \phi} R_{\ell, l}(\rho),
$$

where

$$
R_{\ell, l}(\rho)=N_{\ell, l} \xi^{(\ell-l) / 2} e^{-\xi / 2} L_{l}^{\ell-l}(\xi) .
$$

Here we have used the definitions $N_{\ell, l}=\left(B_{e} l ! / \ell !\right)^{1 / 2}$ and $\xi=B_{e} \rho^{2} / 2$, while $L_{m}^{\alpha}(x)$ are the associated Laguerre polynomials. It can be seen that the functions $W_{\bar{p}}^{s}(x)$ satisfy the orthogonality relations

$$
\int d^{3} r W_{\bar{p}^{\prime}}^{s}(x)^{*} W_{\bar{p}}^{s}(x)=(2 \pi)^{3} \delta_{\ell \ell^{\prime}} \delta_{u^{\prime}} \delta\left(p_{z}-p_{z}^{\prime}\right) .
$$


In addition, the creation and annihilation operators in Eq. (1) satisfy the commutation relations

$$
\begin{aligned}
{\left[a^{\sigma}(\breve{p}), a^{\sigma^{\prime}}\left(\breve{p}^{\prime}\right)^{\dagger}\right] } & =2 E_{\pi^{\sigma}}(2 \pi)^{3} \delta_{\ell \ell^{\prime}} \delta_{u^{\prime}} \delta\left(p_{z}-p_{z}^{\prime}\right) \delta_{\sigma \sigma^{\prime}}, \\
{\left[a^{\sigma}(\breve{p}), a^{\sigma^{\prime}}\left(\breve{p}^{\prime}\right)\right] } & =0 .
\end{aligned}
$$

Note that with these conventions the operators $a^{\sigma}(\breve{p})$ turn out to have different dimensions from the creation and annihilation operators that are usually defined in absence of the external magnetic field.

It is also useful to calculate the particle number associated with the state $\left|\pi^{\sigma}(\breve{p})\right\rangle=a^{\sigma}(\breve{p})^{\dagger}|0\rangle$ in a volume $V$. Given our gauge choice, it is convenient to consider an infinite area in the $x y$ plane and a finite length $L$ along the $z$ axis. We obtain

$$
\begin{aligned}
n_{\pi^{\sigma}} & =\int_{V} d^{3} r\left\langle\pi^{\sigma}(\breve{p})\left|i\left[\phi_{\pi^{\sigma}}^{s} \partial_{t} \phi_{\pi^{\sigma}}^{s}-\left(\partial_{t} \phi_{\pi^{\sigma}}^{s} \dagger\right) \phi_{\pi^{\sigma}}^{s}\right]\right| \pi^{\sigma}(\breve{p})\right\rangle \\
& =2 E_{\pi^{\sigma}}(2 \pi)^{2} L .
\end{aligned}
$$

Note that we are normalizing to $8 \pi^{2} E$ particles per unit length, which differs from the usual normalization $\rho=n / V=2 E$.

\section{B. Charged lepton field}

Assuming the same conventions for the magnetic field and considering the symmetric gauge, for the charged lepton fields we have

$$
\begin{aligned}
\psi_{l}^{s}(x)= & \sum_{\tau=1,2} \sum_{n, v=0}^{\infty} \int \frac{d q_{z}}{(2 \pi)^{3} 2 E_{l}} \\
& \times\left[b(\breve{q}, \tau) U_{l}^{s}(x, \breve{q}, \tau)+d(\breve{q}, \tau)^{\dagger} V_{l}^{-s}(x, \breve{q}, \tau)\right],
\end{aligned}
$$

where $\breve{q}=\left(n, v, q_{z}\right), E_{l}=\sqrt{m_{l}^{2}+2 n B_{e}+q_{z}^{2}}$ and $s=$ $\operatorname{sign}\left(Q_{l} B\right)$, with $Q_{l}=-|e|$ (thus, $\left.B_{e}=\left|Q_{l} B\right|\right)$. Here, $q_{z}$ is an arbitrary real number, while $n$ and $v$ are non-negative integer numbers. The creation and annihilation operators satisfy

$$
\begin{aligned}
\left\{b(\breve{q}, \tau), b\left(\breve{q}^{\prime}, \tau^{\prime}\right)^{\dagger}\right\} & =\left\{d(\breve{q}, \tau), d\left(\breve{q}^{\prime}, \tau^{\prime}\right)^{\dagger}\right\} \\
& =2 E_{l}(2 \pi)^{3} \delta_{\tau \tau^{\prime}} \delta_{n n^{\prime}} \delta_{v v^{\prime}} \delta\left(q_{z}-q_{z}^{\prime}\right), \\
\left\{b(\breve{q}, \tau), d\left(\breve{q}^{\prime}, \tau^{\prime}\right)^{\dagger}\right\} & =\left\{d(\breve{q}, \tau)^{\dagger}, b\left(\breve{q}^{\prime}, \tau^{\prime}\right)^{\dagger}\right\}=0 .
\end{aligned}
$$

For $n>0$ the spinors in Eq. (8) are given, in the Weyl basis, by

$$
\begin{gathered}
U_{l}^{s}(x, \breve{q}, \tau)=\frac{\sqrt{\pi}}{\sqrt{E_{l}+m_{l}}} e^{-i\left(E_{l} t-q_{z} r_{z}\right)} e^{-i s(n-v-1 / 2) \phi}\left[\delta_{\tau, 1}\left(\begin{array}{c}
e^{-i \phi / 2} \varepsilon_{-} R_{n_{s+}, v}(\rho) \\
i s e^{i \phi / 2} \sqrt{2 n B_{e}} R_{n_{s-}, v}(\rho) \\
e^{-i \phi / 2} \varepsilon_{+} R_{n_{s+}, v}(\rho) \\
-i s e^{i \phi / 2} \sqrt{2 n B_{e}} R_{n_{s-}, v}(\rho)
\end{array}\right)+\delta_{\tau, 2}\left(\begin{array}{c}
-i s e^{-i \phi / 2} \sqrt{2 n B_{e}} R_{n_{s+}, v}(\rho) \\
e^{i \phi / 2} \varepsilon_{+} R_{n_{s-}, v}(\rho) \\
i s e^{-i \phi / 2} \sqrt{2 n B_{e}} R_{n_{s+}, v}(\rho) \\
e^{i \phi / 2} \varepsilon_{-} R_{n_{s-}, v}(\rho)
\end{array}\right)\right], \\
V_{l}^{-s}(x, \breve{q}, \tau)=\frac{\sqrt{\pi}}{\sqrt{E_{l}+m_{l}}} e^{i\left(E_{l} t-q_{z} r_{z}\right)} e^{-i s(n-v-1 / 2) \phi}\left[\delta_{\tau, 1}\left(\begin{array}{c}
i s e^{-i \phi / 2} \sqrt{2 n B_{e}} R_{n_{s+}, v}(\rho) \\
e^{i \phi / 2} \varepsilon_{+} R_{n_{s-}, v}(\rho) \\
i s e^{-i \phi / 2} \sqrt{2 n B_{e}} R_{n_{s+}, v}(\rho) \\
-e^{i \phi / 2} \varepsilon_{-} R_{n_{s-}, v}(\rho)
\end{array}\right)+\delta_{\tau, 2}\left(\begin{array}{c}
-e^{-i \phi / 2} \varepsilon_{-} R_{n_{s+}, v}(\rho) \\
i s e^{i \phi / 2} \sqrt{2 n B_{e}} R_{n_{s-}, v}(\rho) \\
e^{-i \phi / 2} \varepsilon_{+} R_{n_{s+}, v}(\rho) \\
i s e^{i \phi / 2} \sqrt{2 n B_{e} R_{n_{s-}, v}}(\rho)
\end{array}\right)\right]
\end{gathered}
$$

where $\varepsilon_{ \pm}=E_{l}+m_{l} \pm q_{z}$. Here the non-negative integer index $n_{s \lambda}$ is related to the quantum number $n$ by $n_{s \pm}=$ $n-(1 \mp s) / 2$.

In the particular case of the lowest Landau level (LLL) $n=0$, only one polarization is allowed. Using the notation $\breve{q}_{L L L}=\left(0, v, p_{z}\right)$, the explicit form of the spinors in this case are

$$
U_{l}^{s}\left(x, \breve{q}_{L L L}\right)=\frac{\sqrt{\pi}}{\sqrt{E_{l}+m_{l}}} e^{-i\left(E_{l} t-q_{z} r_{z}\right)} e^{i s v \phi} R_{0, v}(\rho)\left[\delta_{s, 1}\left(\begin{array}{c}
\varepsilon_{-} \\
0 \\
\varepsilon_{+} \\
0
\end{array}\right)+\delta_{s,-1}\left(\begin{array}{c}
0 \\
\varepsilon_{+} \\
0 \\
\varepsilon_{-}
\end{array}\right)\right]
$$




$$
V_{l}^{-s}\left(x, \breve{q}_{L L L}\right)=\frac{\sqrt{\pi}}{\sqrt{E_{l}+m_{l}}} e^{i\left(E_{l} t-q_{z} r_{z}\right)} e^{i s v \phi} R_{0, v}(\rho)\left[\delta_{s, 1}\left(\begin{array}{c}
-\varepsilon_{-} \\
0 \\
\varepsilon_{+} \\
0
\end{array}\right)+\delta_{s,-1}\left(\begin{array}{c}
0 \\
\varepsilon_{+} \\
0 \\
-\varepsilon_{-}
\end{array}\right)\right]
$$

It can be seen that the spinors satisfy the orthogonality relations

$$
\begin{aligned}
\int d^{3} r U^{s}(x, \breve{q}, \tau)^{\dagger} U^{s}\left(x, \breve{q}^{\prime}, \tau^{\prime}\right) & =\int d^{3} r V^{-s}(x, \breve{q}, \tau)^{\dagger} V^{-s}\left(x, \breve{q}^{\prime}, \tau^{\prime}\right)=2 E_{l}(2 \pi)^{3} \delta_{\breve{q}, \breve{q}^{\prime}} \delta_{\tau \tau^{\prime}}, \\
\int d^{3} r U^{s}(x, \breve{q}, \tau)^{\dagger} V^{-s}\left(x, \breve{q}^{\prime}, \tau^{\prime}\right) & =\int d^{3} r V^{-s}(x, \breve{q}, \tau)^{\dagger} U^{s}\left(x, \breve{q}^{\prime}, \tau^{\prime}\right)=0, \\
\int d^{3} r \bar{U}^{s}(x, \breve{q}, \tau) U^{s}\left(x, \breve{q}^{\prime}, \tau^{\prime}\right) & =-\int d^{3} r \bar{V}^{-s}(x, \breve{q}, \tau) V^{-s}\left(x, \breve{q}^{\prime}, \tau^{\prime}\right)=2 m_{l}(2 \pi)^{3} \delta_{\breve{q}, \breve{q}^{\prime}} \delta_{\tau \tau^{\prime}}, \\
\int d^{3} r \bar{U}^{s}(x, \breve{q}, \tau) V^{-s}\left(\tilde{x}, \breve{q}^{\prime}, \tau^{\prime}\right) & =\int d^{3} r \bar{V}^{-s}(x, \breve{q}, \tau) U^{s}\left(\tilde{x}, \breve{q}^{\prime}, \tau^{\prime}\right)=0,
\end{aligned}
$$

which are valid for both $n=0$ and $n>0$. We have used here the definitions $\delta_{\breve{q}, \breve{q}^{\prime}}=\delta_{n n^{\prime}} \delta_{v v^{\prime}} \delta\left(q_{z}-q_{z}^{\prime}\right)$ and $\tilde{x}=(t,-\vec{r})$.

An alternative representation of the spinors in Eq. (8), closer to the Ritus representation often used in the Landau gauge, is given in Appendix A.

\section{Commutation relations and quantum numbers in the symmetric gauge}

In this subsection we discuss some properties of the operators and particle states. We consider first the case of charged leptons. We recall that, in this case, if a physical quantity has an associated quantum mechanical operator $\mathcal{O}$, the field theoretical realization of this operator is given by

$$
\tilde{\mathcal{O}}=\int_{V} d^{3} r: \psi_{l}^{s}(x)^{\dagger} \mathcal{O} \psi_{l}^{s}(x): .
$$

Let us recall that the Dirac Hamiltonian for a charged particle in a uniform magnetic field is given by

$$
H=\gamma^{0}[\vec{\gamma} \cdot \vec{P}+m] .
$$

Here $\vec{P}$ is the mechanical momentum, related to the canonical momentum $\vec{p}=-i \vec{\nabla}$ by

$$
\vec{P}=\vec{p}-Q_{l} \vec{A},
$$

where $\vec{A}$ is the vector potential associated to the uniform magnetic field $\vec{B}$. Although the explicit relation between both momenta depends on the gauge choice, it is seen that $\vec{P}$ is a gauge covariant quantity. For a magnetic field orientated along the $z$ direction, the relation

$$
\left[\tilde{P}_{j}, \tilde{P}_{k}\right]=i B\left(\delta_{j 1} \delta_{k 2}-\delta_{j 2} \delta_{k 1}\right) \tilde{Q}
$$

is found to be satisfied (integer indices 1,2,3 are intended to be equivalent to $x, y, z$ ). Here,

$$
\tilde{Q}=Q_{l} \sum_{\tau=1,2} \sum_{n, v=0}^{\infty} \int \frac{d q_{z}}{(2 \pi)^{3}}\left[b(\breve{q}, \tau)^{\dagger} b(\breve{q}, \tau)-d(\breve{q}, \tau)^{\dagger} d(\breve{q}, \tau)\right] .
$$

Using the spinors defined in the previous subsection, a straightforward calculation shows that, as expected, in the symmetric gauge one gets

$\tilde{H}|l(\breve{q}, \tau)\rangle=E_{l}|l(\breve{q}, \tau)\rangle, \quad \tilde{H}|\bar{l}(\breve{q}, \tau)\rangle=E_{l}|\bar{l}(\breve{q}, \tau)\rangle$,

where $E_{l}=\sqrt{m_{l}^{2}+2 n B_{e}+q_{z}^{2}}$.

We introduce now the canonical orbital angular momentum operator $\vec{l}=\vec{r} \times \vec{p}$ and the spin operator $\vec{S}$. Given the fact that the magnetic field breaks the rotational invariance, only the components of these operators along the $z$ axis are relevant. Using cylindrical coordinates, the $z$ components $l_{z}$ and $S_{z}$ are given by

$$
l_{z}=-i \frac{\partial}{\partial \phi}, \quad S_{z}=\frac{1}{2} \operatorname{diag}(1,-1,1,-1) .
$$

Defining the canonical total angular momentum as $j_{z}=l_{z}+S_{z}$ and using the spinors defined in the previous subsection we obtain

$\tilde{j}_{z}|l(\breve{q}, \tau)\rangle=j_{z}^{(l)}|l(\breve{q}, \tau)\rangle, \quad \tilde{j}_{z}|\bar{l}(\breve{q}, \tau)\rangle=-j_{z}^{(l)}|\bar{l}(\breve{q}, \tau)\rangle$,

with

$$
j_{z}^{(l)}=-s(n-v-1 / 2) .
$$


Thus, as expected, we see that for the charged leptons in the symmetric gauge one can find energy eigenstates that are also eigenstates of the $z$ component of the total canonical angular momentum. Since the energy eigenvalues do not depend on $v$, we see that, in the symmetric gauge, a state of a given Landau level $n$ is in general a linear combination of an infinite set of degenerate states with well-defined quantum number $j_{z}^{(l)}$. This is consistent with the fact that, in this gauge, one has $\left[\tilde{j}_{z}, \tilde{H}\right]=0$, as can be verified from the previously given expressions for $H$ and $j_{z}$. We stress here that $j_{z}$ is not a gauge covariant quantity. In the Landau gauge, for example, it is not difficult to check that $\tilde{j}_{z}$ does not commute with $\tilde{H}$, therefore in general it is not expected to be conserved. Turning back to the symmetric gauge, it is worth noticing that only the canonical total angular momentum is well defined, i.e., energy eigenstates are not in general eigenstates of $\tilde{l}_{z}$ and $\tilde{S}_{z}$ separately.

Associated to the mechanical momentum $\vec{P}$ we can define the mechanical orbital angular momentum $\vec{L}=$ $\vec{r} \times \vec{P}$ and the mechanical total angular momentum $\vec{J}=\vec{L}+\vec{S}$. In the same way as $\vec{P}, \vec{J}$ is a gauge covariant operator. An explicit calculation shows, however, that for a magnetic field along the $z$-axis one has

$$
\left[\tilde{J}_{z}, \tilde{H}\right]=-i s B_{Q} \int d^{3} r \bar{\psi}(x)\left(r_{x} \gamma^{1}+r_{y} \gamma^{2}\right) \psi(x),
$$

which is valid in any gauge. Hence, $J_{z}$ is a gauge covariant quantity but it is not a conserved quantity. In particular, an explicit calculation in the symmetric gauge shows that $\tilde{J}_{z}$ is not diagonal in the basis of energy eigenstates.

Let us consider now the limit in which the charged lepton mass $m_{l}$ vanishes. This is interesting when the magnetic field is relatively strong, say $B_{e} \gg m_{l}^{2}$. In the limit $m_{l}=0$ the chirality operator $\gamma_{5}$ becomes equivalent to the helicity operator $\hat{P} \cdot \vec{S}$, and commutes with $H$. Consequently, one can obtain energy eigenstates of well defined chirality/ helicity. For arbitrary $n>0$ they can be constructed as linear combinations of the two polarization states. We get

$$
|l(\breve{q}, L)\rangle_{\mathrm{ch}}=-i \frac{\sqrt{E_{l}-q_{z}}}{\sqrt{2 E_{l}}}|l(\breve{q}, 1)\rangle_{\mathrm{ch}}+\frac{\sqrt{E_{l}+q_{z}}}{\sqrt{2 E_{l}}}|l(\breve{q}, 2)\rangle_{\mathrm{ch}},
$$

$$
|l(\breve{q}, R)\rangle_{\mathrm{ch}}=i \frac{\sqrt{E_{l}+q_{z}}}{\sqrt{2 E_{l}}}|l(\breve{q}, 1)\rangle_{\mathrm{ch}}+\frac{\sqrt{E_{l}-q_{z}}}{\sqrt{2 E_{l}}}|l(\breve{q}, 2)\rangle_{\mathrm{ch}} .
$$

(subindices ch indicate that the chiral limit $m_{l} \rightarrow 0$ has been taken). On the other hand, as mentioned above, for the LLL only one polarization is available. Thus, the states associated with the spinors that result from taking $m_{l}=0$ in Eqs. (13) and (14) are already helicity eigenstates. We get

$$
\begin{aligned}
& \left.\tilde{\gamma}_{5}\left|l\left(\left(\breve{q}_{L L L}\right)\right\rangle_{\mathrm{ch}}=s \operatorname{sign}\left(q_{z}\right)\right| l\left(\breve{q}_{L L L}\right)\right\rangle_{\mathrm{ch}}, \\
& \left.\tilde{\gamma}_{5}\left|\bar{l}\left(\left(\breve{q}_{L L L}\right)\right\rangle_{\mathrm{ch}}=-s \operatorname{sign}\left(q_{z}\right)\right| \bar{l}\left(\breve{q}_{L L L}\right)\right\rangle_{\mathrm{ch}} .
\end{aligned}
$$

This implies that for large enough magnetic fields-such that only the LLL is relevant and $m_{l}$ can be neglecteda negatively charged lepton (like the muon or the electron) is lefthanded if $B$ and $q_{z}$ are either both positive or both negative, and it is righthanded otherwise.

We briefly consider now the pion eingenstates. Their angular momentum can be analyzed following similar steps as before. For the canonical orbital angular momentum we get

$$
\tilde{l}_{z}\left|\pi^{\sigma}(\breve{p})\right\rangle=l_{z}^{\left(\pi^{\sigma}\right)}\left|\pi^{\sigma}(\breve{p})\right\rangle
$$

with

$$
l_{z}^{\left(\pi^{\sigma}\right)}=-s(\ell-\imath)
$$

where $s=\operatorname{sign}(\sigma B)$. As in the case of charged leptons, the mechanical angular momentum $L_{z}$ is not a conserved quantity, thus, it is not diagonal in the basis of energy eigenstates.

\section{CALCULATION OF THE $\pi^{-} \rightarrow l \bar{\nu}_{l}$ DECAY WIDTH IN THE PRESENCE OF AN EXTERNAL MAGNETIC FIELD USING THE SYMMETRIC GAUGE}

Let us analyze the decay width for the process $\pi^{-} \rightarrow l \bar{\nu}_{l}$, with $l=\mu^{-}, e^{-}$, in the presence of a uniform magnetic field using the symmetric gauge. For definiteness we will take $B>0$, i.e., $s=-1$. Following the notation introduced in the previous section, the initial charged pion state is determined by the quantum numbers $\breve{p}=\left(\ell, l, p_{z}\right)$, the associated energy being $E_{\pi^{-}}=\sqrt{m_{\pi^{-}}^{2}+(2 \ell+1) B_{e}+p_{z}^{2}}$. The quantum numbers corresponding to the outgoing lepton state are taken to be $\breve{q}=\left(n, v, q_{z}\right)$, together with a polarization index $\tau$. In this case the energy is given by $E_{l}=\sqrt{m_{l}^{2}+2 n B_{e}+q_{z}^{2}}$. Finally, being electrically neutral, the outgoing antineutrino is taken to be in the cylindrical basis discussed in Appendix B. Thus, the associated quantum numbers are $\breve{k}=\left(J, k_{\perp}, k_{z}\right)$, where $J$ is an integer while $k_{\perp}$ and $k_{z}$ are real numbers, with $k_{\perp}>0$. The corresponding energy is $E_{\bar{\nu}_{l}}=\sqrt{k_{\perp}^{2}+k_{z}^{2}}$.

\section{A. The decay amplitude}

Using the notation introduced in the previous section, the transition matrix element for the process we are interested in is given by 


$$
\begin{aligned}
& \left\langle l(\breve{q}, \tau) \bar{\nu}_{l}(\breve{k}, R)\left|\mathcal{L}_{W}\right| \pi^{-}(\breve{p})\right\rangle \\
& =-\frac{G_{F}}{\sqrt{2}} \cos \theta_{c} \\
& \quad \times \int d^{4} x H_{L}^{-, \mu}(x, \breve{p}) \bar{U}_{l}^{-}(x, \breve{q}, \tau) \gamma_{\mu}\left(1-\gamma_{5}\right) V_{\nu_{l}}(x, \breve{k}, R),
\end{aligned}
$$

where $H_{L}^{-, \mu}(x, \breve{p})$ stands for the matrix element of the hadronic current,

$$
\begin{aligned}
H_{L}^{-, \mu}(x, \breve{p}) & =H_{V}^{-, \mu}(x, \breve{p})-H_{A}^{-, \mu}(x, \breve{p}) \\
& =\left\langle 0\left|\bar{\psi}_{u}(x) \gamma^{\mu}\left(1-\gamma_{5}\right) \psi_{d}(x)\right| \pi^{-}(\breve{p})\right\rangle .
\end{aligned}
$$

Following the definitions and conventions of Ref. [22], the hadronic matrix elements can be parametrized as

$$
\begin{aligned}
H_{\|, L}^{-, \epsilon} & =H_{L}^{-, 0}+\epsilon H_{L}^{-, 3} \\
& =\sqrt{2}\left(\epsilon f_{\pi^{-}}^{(V)}-f_{\pi^{-}}^{(A 1)}\right)\left(\mathcal{D}^{0}+\epsilon \mathcal{D}^{3}\right) W_{\bar{p}}^{-}(x), \\
H_{\perp, L}^{-, \epsilon} & =H_{L}^{-, 1}+i \epsilon H_{L}^{-, 2} \\
& =-\sqrt{2}\left(f_{\pi^{-}}^{(A 1)}+\epsilon f_{\pi^{-}}^{(A 2)}-f_{\pi^{-}}^{(A 3)}\right)\left(\mathcal{D}^{1}+i \epsilon \mathcal{D}^{2}\right) W_{\bar{p}}^{-}(x) .
\end{aligned}
$$

Rewriting $H_{L}^{-, \mu}$ in terms of the parallel and perpendicular components one gets $\gamma_{\mu}\left(1-\gamma_{5}\right) H_{L}^{-, \mu}(x, \breve{p})=\left(\begin{array}{cccc}0 & 0 & 0 & 0 \\ 0 & 0 & 0 & 0 \\ H_{\|, L}^{-,+} & H_{\perp, L}^{-,-} & 0 & 0 \\ H_{\perp, L}^{-,+} & H_{\|, L}^{-,-} & 0 & 0\end{array}\right)$,

with

$$
\begin{aligned}
H_{\|, L}^{-, \epsilon}= & -i \sqrt{2}\left(\epsilon f_{\pi^{-}}^{(V)}-f_{\pi^{-}}^{(A 1)}\right)\left(E_{\pi^{-}}+\epsilon p_{z}\right) W_{\bar{p}}^{-}(x), \\
H_{\perp, L}^{-, \epsilon}= & -\epsilon \sqrt{2}\left(f_{\pi^{-}}^{(A 1)}+\epsilon f_{\pi^{-}}^{(A 2)}-f_{\pi^{-}}^{(A 3)}\right) \sqrt{(2 \ell+1+\epsilon) B_{e}} \\
& \times W_{\bar{p}+\epsilon}^{-}(x),
\end{aligned}
$$

where $\bar{p}+\epsilon=\left(E_{\pi^{-}}, \ell+\epsilon, l, p_{z}\right)$.

Using these expressions together with the explicit form of the charged lepton and antineutrino spinors (see Sec. II B and Appendix B) we get

$$
\begin{aligned}
& \left\langle l(\breve{q}, \tau) \bar{\nu}_{l}(\breve{k}, R)\left|\mathcal{L}_{W}\right| \pi^{-}(\breve{p})\right\rangle \\
& =(2 \pi)^{3} \delta\left(E_{\pi^{-}}-E_{l}-E_{\bar{\nu}_{l}}\right) \delta\left(p_{z}-q_{z}-k_{z}\right) \\
& \quad \times \delta_{\ell-l, n-v+J^{-1}} \mathcal{M}(\breve{p}, \breve{q}, \breve{k}, \tau),
\end{aligned}
$$

where

$$
\begin{aligned}
& \mathcal{M}(\breve{p}, \breve{q}, \breve{k}, 1)=-\sqrt{2} G_{F} \cos \theta_{c} \frac{(-i)^{J+1}}{\sqrt{E_{l}+m_{l}}}\left[\left(E_{l}+m_{l}-q_{z}\right) A(\breve{p}, \breve{q}, \breve{k})+\sqrt{2 n B_{e}} B(\breve{p}, \breve{q}, \breve{k})\right], \\
& \mathcal{M}(\breve{p}, \breve{q}, \breve{k}, 2)=\sqrt{2} G_{F} \cos \theta_{c} \frac{(-i)^{J}}{\sqrt{E_{l}+m_{l}}}\left[\sqrt{2 n B_{e}} A(\breve{p}, \breve{q}, \breve{k})+\left(E_{l}+m_{l}+q_{z}\right) B(\breve{p}, \breve{q}, \breve{k})\right] .
\end{aligned}
$$

Here we have used the definitions

$$
\begin{aligned}
& A(\breve{p}, \breve{q}, \breve{k})=a_{\pi^{-}}\left(E_{\pi^{-}}+p_{z}\right) \sqrt{E_{\bar{\nu}_{l}}-k_{z}} I_{1}-d_{\pi^{-}} \sqrt{2 \ell B_{e}} \sqrt{E_{\bar{\nu}_{l}}+k_{z}} I_{2}, \\
& B(\breve{p}, \breve{q}, \breve{k})=b_{\pi^{-}}\left(E_{\pi^{-}}-p_{z}\right) \sqrt{E_{\bar{\nu}_{l}}+k_{z}} I_{4}-c_{\pi^{-}} \sqrt{2(\ell+1) B_{e}} \sqrt{E_{\bar{\nu}_{l}}-k_{z}} I_{3},
\end{aligned}
$$

and

$$
\begin{array}{ll}
a_{\pi^{-}}=f_{\pi^{-}}^{(A 1)}-f_{\pi^{-}}^{(V)}, & b_{\pi^{-}}=f_{\pi^{-}}^{(A 1)}+f_{\pi^{-}}^{(V)}, \\
c_{\pi^{-}}=f_{\pi^{-}}^{(A 1)}+f_{\pi^{-}}^{(A 2)}-f_{\pi^{-}}^{(A 3)}, & d_{\pi^{-}}=f_{\pi^{-}}^{(A 1)}-f_{\pi^{-}}^{(A 2)}-f_{\pi^{-}}^{(A 3)},
\end{array}
$$

while $I_{i}, i=1, \ldots 4$ are radial integrals given by

$$
\begin{array}{ll}
I_{1}=\mathcal{I}(\ell, l, n-1, v), & I_{2}=\mathcal{I}(\ell-1, l, n-1, v), \\
I_{3}=\mathcal{I}(\ell+1, l, n, v), & I_{4}=\mathcal{I}(\ell, l, n, v),
\end{array}
$$

where

$$
\begin{aligned}
& \mathcal{I}(\ell, l, n, v) \\
& =2 \pi \int_{0}^{\infty} d \rho \rho R_{\ell, l}(\rho) R_{n, v}(\rho) J_{(\ell-l)-(n-v)}\left(k_{\perp} \rho\right) .
\end{aligned}
$$

The evaluation of these integrals for arbitrary particle states is given in Appendix C.

Note that, due to the $\delta$ functions appearing in Eq. (38), not all the variables in the expressions given in Eqs. (39) are 
independent. While the first two $\delta$ functions correspond to the conservation of the energy and the $z$ component of the linear momentum, the last one leads to the relation $\ell-\imath=n-v-1 / 2+\jmath-1 / 2$. Taking into account that for antineutrinos (in our basis) one has $j_{z}^{\left(\bar{\nu}_{l}\right)}=J-1 / 2$ (see Appendix B), using Eqs. (24) and (30), and recalling that we are considering $s=-1$, this relation can be written as $j_{z}^{\left(\pi^{-}\right)}=j_{z}^{(l)}+j_{z}^{\left(\bar{\nu}_{l}\right)}$. Therefore, the last $\delta$ function in Eq. (38) implies that, as expected, the $z$ component of the canonical total angular momentum is conserved in the decay process when the symmetric gauge is used.

Now let us concentrate on the situation in which the decaying pion is in the lowest energy state (LES). This corresponds to $\ell=0$ and $p_{z}=0$, i.e., $\breve{p}_{\mathrm{LES}}=(0, \imath, 0)$. In this case the radial integrals $I_{i}$ get simplified. Introducing $x=k_{\perp}^{2} /\left(2 B_{e}\right)$, we get

$$
\begin{aligned}
& I_{1}^{(\ell=0)}=2 \pi(-1)^{n-1} \frac{x^{(n-1) / 2}}{\sqrt{(n-1) !}} F_{l, v}(x), \\
& I_{2}^{(\ell=0)}=0, \\
& I_{3}^{(\ell=0)}=2 \pi(-1)^{n} \frac{x^{(n-1) / 2}}{\sqrt{n !}}(x-n) F_{l, v}(x), \\
& I_{4}^{(\ell=0)}=2 \pi(-1)^{n} \frac{x^{n / 2}}{\sqrt{n !}} F_{l, v}(x),
\end{aligned}
$$

where

$$
F_{l, v}(x)=\sqrt{\frac{l !}{v !}}(-1)^{l+v} x^{(v-l) / 2} e^{-x} L_{l}^{v-l}(x) .
$$

The decay amplitudes simplify to

$$
\begin{aligned}
\mathcal{M}\left(\breve{p}_{\mathrm{LES}}, \breve{q}, \breve{k}, 1\right)= & G_{F} \cos \theta_{c}(-1)^{n}(-i)^{J+1} 2 \pi \sqrt{\frac{2\left(E_{\bar{\nu}_{l}}-k_{z}\right)}{E_{l}+m_{l}}} \frac{x^{(n-1) / 2}}{\sqrt{n-1 !}} F_{l, v}(x) \\
& \times\left[a_{\pi^{-}} E_{\pi^{-}}\left(E_{l}+m_{l}-q_{z}\right)-b_{\pi^{-}} E_{\pi^{-}}\left(E_{\bar{\nu}_{l}}+k_{z}\right)+c_{\pi^{-}} 2 B_{e}(x-n)\right], \\
\mathcal{M}\left(\breve{p}_{\mathrm{LES}}, \breve{q}, \breve{k}, 2\right)= & G_{F} \cos \theta_{c}(-1)^{n+1}(-i)^{J} 2 \pi \sqrt{\frac{E_{\bar{\nu}_{l}}-k_{z}}{B_{e}\left(E_{l}+m_{l}\right)}} \frac{x^{(n-1) / 2}}{\sqrt{n !}} F_{l, v}(x) \\
& \times\left\{a_{\pi^{-}} E_{\pi^{-}} 2 n B_{e}-\left(E_{l}+m_{l}+q_{z}\right)\left[b_{\pi^{-}} E_{\pi^{-}}\left(E_{\bar{\nu}_{l}}+k_{z}\right)-c_{\pi^{-}} 2 B_{e}(x-n)\right]\right\} .
\end{aligned}
$$

It is interesting at this point to consider the situation in which the magnetic field is large enough so that the outgoing charged lepton can only be in the lowest Landau level. As mentioned in the previous section, only one polarization state is allowed in this case. Since we are considering $s=-1$, this corresponds to $\tau=2$. We get

$$
\begin{aligned}
\mathcal{M}\left(\breve{p}_{\mathrm{LES}}, \breve{q}_{L L L}, \breve{k}\right) & \\
= & G_{F} \cos \theta_{c}(-i)^{\jmath} 2 \pi \sqrt{\frac{2}{E_{l}+m_{l}}} F_{l, v}(x) \\
& \times\left(E_{l}+m_{l}+q_{z}\right) \sqrt{E_{\nu_{l}}+k_{z}}\left[b_{\pi^{-}} E_{\pi^{-}}-c_{\pi^{-}}\left(E_{\nu_{l}}-k_{z}\right)\right],
\end{aligned}
$$

with $\breve{q}_{L L L}=\left(0, v, q_{z}\right)$. We remark that, due to the $\delta$ functions in Eq. (38), the relations $q_{z}=-k_{z}, \jmath=v-\imath+1$ and $k_{z}= \pm \sqrt{\left(E_{\pi^{-}}^{2}+2 B_{e} x-m_{l}^{2}\right)^{2}-8 B_{e} E_{\pi^{-}}^{2} x} /\left(2 E_{\pi^{-}}\right)$are satisfied. Therefore, recalling that $x=k_{\perp}^{2} /\left(2 B_{e}\right)$, we see that for fixed $E_{\pi^{-}}$and given definite values of $l$ and $v$ the amplitude is a function of $k_{\perp}$. Contrary to the claim in Ref. [9], we conclude that by no means angular momentum conservation implies that $k_{\perp}$ has to be zero. In fact, one should integrate over the full range of values of $k_{\perp}$ from zero to infinity to calculate the total width.

A final observation concerns the situation in which $B_{e} \gg m_{l}^{2}$. In this case, we can neglect the charged lepton mass in Eq. (48), obtaining

$$
\begin{aligned}
\mathcal{M} & \left(\breve{p}_{\mathrm{LES}}, \breve{q}_{L L L}, \breve{k}\right)_{\mathrm{ch}} \\
= & G_{F} \cos \theta_{c}(-i)^{\jmath} 2 \pi \sqrt{2 E_{l}} F_{l, v}(x) \\
& \times\left[1-\operatorname{sign}\left(k_{z}\right)\right] \sqrt{E_{\nu_{l}}+k_{z}}\left[b_{\pi^{-}} E_{\pi^{-}}-c_{\pi^{-}}\left(E_{\nu_{l}}-k_{z}\right)\right],
\end{aligned}
$$

where we have used that in the present case $E_{l}=\left|k_{z}\right|$. As seen, while for $k_{z}>0$ the amplitude vanishes, for $k_{z}<0$ in general it does not. This can be understood in terms of helicity conservation. As discussed in Sec. II C, in the limit $m_{l} \rightarrow 0$ for a charged lepton in the LLL we have (recall once again that we are considering $s=-1$ )

$$
\gamma_{5}\left|l\left(\breve{q}_{L L L}\right)\right\rangle_{\mathrm{ch}}=-\operatorname{sign}\left(q_{z}\right)\left|l\left(\breve{q}_{L L L}\right)\right\rangle_{\mathrm{ch}} .
$$

Noting that $q_{z}=-k_{z}$, we see that for $k_{z}>0$ the outgoing charged lepton would be righthanded, which is forbidden 
by helicity conservation since antineutrinos are always righthanded. This is very different from what happens in the absence of a magnetic field. For $B=0$, helicity conservation implies that the total decay amplitude of a pion at rest must vanish as $m_{l} \rightarrow 0$. At large magnetic field, however, it only implies that the projection of the antineutrino momentum in the direction of $\vec{B}$ must be opposite to $\vec{B}$.

\section{B. Decay width}

On general grounds, the decay width for the process we are interested in is given by

$$
\begin{aligned}
\Gamma_{l}^{-}(B)= & \lim _{L, T \rightarrow \infty} \sum_{\tau=1,2} \sum_{n, v, j=0}^{\infty} \int \frac{d q_{z}}{(2 \pi)^{3} 2 E_{l}} \frac{d k_{z} d k_{\perp} k_{\perp}}{(2 \pi)^{2} 2 E_{\bar{\nu}_{l}}} \\
& \times \frac{\left|\left\langle l(\breve{q}, \tau) \bar{\nu}_{l}(\breve{k}, R)\left|\mathcal{L}_{W}\right| \pi^{-}(\breve{p})\right\rangle\right|^{2}}{2(2 \pi)^{2} E_{\pi^{-}} L T}
\end{aligned}
$$

where $T$ and $L$ are the time interval and length on the $z$-axis in which the interaction is active. At the end of the calculation, the limit $L, T \rightarrow \infty$ should be taken. Using Eq. (38) we get

$$
\begin{aligned}
\Gamma_{l}^{-}(B)= & \frac{1}{16 \pi E_{\pi^{-}}} \sum_{n, v, J=0}^{\infty} \int \frac{d q_{z} d k_{z} d k_{\perp} k_{\perp}}{(2 \pi)^{2} E_{l} E_{\bar{\nu}_{l}}} \\
& \times \delta\left(E_{\pi^{-}}-E_{l}-E_{\bar{\nu}_{l}}\right) \delta\left(p_{z}-q_{z}-k_{z}\right) \\
& \times \delta_{\ell-l, n-v-1+\jmath}\left|\mathcal{M}_{\pi^{-} \rightarrow l \bar{l}_{l}}\right|^{2}
\end{aligned}
$$

where

$$
\overline{\left|\mathcal{M}_{\pi^{-} \rightarrow \mid \bar{\nu}_{l}}\right|^{2}} \equiv \sum_{\tau=1,2}|\mathcal{M}(\breve{p}, \breve{q}, \breve{k}, \tau)|^{2}
$$

and the amplitudes for $\tau=1,2$ are given in Eq. (39).
Now, as it is usually done, we concentrate on the situation in which the decaying pion is in the lowest energy state. This corresponds to $\ell=0$ and $p_{z}=0$. Moreover, as it will be shown below, the decay width will not depend on the value of $l$. The expression in Eq. (52) can be worked out, leading to

$$
\begin{aligned}
\Gamma_{l}^{-}(B)= & \frac{1}{16 \pi E_{\pi^{-}}^{2}} \sum_{n=0}^{n^{\max }} \int_{0}^{k_{\perp}^{\max }} \frac{d k_{\perp} k_{\perp}}{2 \pi} \\
& \times \int \frac{d k_{z}}{2 \pi \bar{k}_{z}}\left[\delta\left(k_{z}-\bar{k}_{z}\right)+\delta\left(k_{z}+\bar{k}_{z}\right)\right] \sum_{v=0}^{\infty} \overline{\left|\mathcal{M}_{\pi^{-} \rightarrow \bar{\nu}_{l}}\right|^{2}},
\end{aligned}
$$

where we have used the definitions

$$
\begin{aligned}
n^{\max } & =\frac{m_{\pi^{-}}^{2}-m_{l}^{2}+B_{e}}{2 B_{e}}, \\
k_{\perp}^{\max } & =E_{\pi^{-}}-\sqrt{m_{l}^{2}+2 n B_{e}}, \\
\overline{k_{z}} & =\frac{1}{2 E_{\pi^{-}}} \sqrt{\left[E_{\pi^{-}}^{2}-2 B_{e}(n-x)-m_{l}^{2}\right]^{2}-8 B_{e} E_{\pi^{-}}^{2} x} .
\end{aligned}
$$

In the amplitudes we must take $q_{z}=-k_{z}$ and $J=$ $v+1-n-\imath$. Using Eqs. (46) and (47) we get

$$
\begin{gathered}
\int \frac{d k_{z}}{2 \pi}\left[\delta\left(k_{z}-\bar{k}_{z}\right)+\delta\left(k_{z}+\overline{k_{z}}\right)\right] \sum_{v=0}^{\infty} \overline{\left|\mathcal{M}_{\pi^{-} \rightarrow \mid \bar{\nu}_{l}}\right|^{2}} \\
=16 \pi G_{F}^{2} \cos ^{2} \theta_{c} \frac{x^{n-1}}{n !} A_{n}(x) \sum_{v=0}^{\infty} F_{l, v}(x)^{2}
\end{gathered}
$$

where

$$
\begin{aligned}
A_{\pi^{-}}^{(n)}(x)= & {\left[E_{\pi^{-}}^{2}-2 B_{e}(n-x)-m_{l}^{2}\right]\left[\frac{m_{l}^{2}}{2}\left(n\left|a_{\pi^{-}}\right|^{2}+x\left|b_{\pi^{-}}\right|^{2}\right)+B_{e}(n-x)\left(n\left|a_{\pi^{-}}-c_{\pi^{-}}\right|^{2}+x\left|b_{\pi^{-}}-c_{\pi^{-}}\right|^{2}\right)\right] } \\
& +2 B_{e} x\left\{E_{\pi^{-}}^{2}\left[n\left|a_{\pi^{-}}-b_{\pi^{-}}\right|^{2}-(n-x)\left|b_{\pi^{-}}-c_{\pi^{-}}\right|^{2}\right]+(n-x) m_{l}^{2}\left|c_{\pi^{-}}\right|^{2}\right\} .
\end{aligned}
$$

To proceed we have to evaluate the sum over the charged lepton quantum number $v$ on the right-hand side of Eq. (58). As shown in Appendix D, one gets

$$
\sum_{v=0}^{\infty} F_{l, v}(x)^{2}=e^{-x}
$$

Using this result we arrive at the final expression for the $\pi^{-} \rightarrow l \bar{\nu}_{l}$ decay width, namely

$$
\Gamma_{l}^{-}(B)=\frac{G_{F}^{2} \cos ^{2} \theta_{c}}{2 \pi E_{\pi^{-}}^{2}} B_{e} \sum_{n=0}^{n_{\max }} \int_{0}^{x_{\max }} d x \frac{1}{\overline{k_{z}}} \frac{x^{n-1}}{n !} e^{-x} A_{\pi^{-}}^{(n)}(x) .
$$

Our result agrees exactly with Eq. (52) of Ref. [22], where the calculation was carried out using the Landau gauge. This provides an additional and explicit confirmation of the gauge independence of our expression for the decay width. It should be noted that, since the sum in Eq. (60) turns out 
to be independent of $l$, the width does not depend on the charged pion canonical angular momentum $j_{z}^{\left(\pi^{-}\right)}$. This is to be expected due to the fact that, as mentioned in Sec. III, $j_{z}^{\left(\pi^{-}\right)}$is a gauge dependent quantity.

\section{SUMMARY AND CONCLUSIONS}

In this work we study the decay width $\pi^{-} \rightarrow l \bar{\nu}_{l}$ in the presence of an arbitrary large uniform magnetic field. We use here the symmetric gauge, as an alternative to a previous analysis carried out in Ref. [22] where the Landau gauge was considered. The usage of the symmetric gauge has the advantage of allowing for a better understanding of the consequences of the axial symmetry of the problem, as well as for a better treatment of angular momenta. In our analysis we introduce charged pion and lepton wave functions and spinors in this gauge, using cylindrical coordinates. To study the conservation of angular momentum, we define the canonical $\left(j_{z}\right)$ and mechanical $\left(J_{z}\right)$ total angular momentum operators. We find that, as expected, even though $j_{z}$ is not a gauge covariant quantity, it turns out to be conserved in the symmetric gauge. On the other hand, $J_{z}$ is shown to be gauge covariant but it is not conserved in any gauge. As shown by explicit calculation [see Eq. (41)], the relevant matrix element for the process $\pi^{-} \rightarrow l \bar{\nu}_{l}$ turns out to be proportional to a Kronecker delta which, when expressed in terms of the $j_{z}$ values of the different fields, clearly reflects the conservation of the canonical total angular momentum of the system. Using the symmetric gauge we also obtain an explicit expression for the decay width $\pi^{-} \rightarrow l \bar{\nu}_{l}$ for the case in which the decaying pion lies in its state of minimum energy (i.e., in the lowest Landau level, with zero linear momentum along the direction of the magnetic field). We show that the total width does not depend on the charged pion canonical angular momentum $j_{z}^{\left(\pi^{-}\right)}$, a fact that is to be expected since $j_{z}^{\left(\pi^{-}\right)}$is a gauge dependent quantity. Moreover, it is seen that the total width is obtained after integrating over the perpendicular momenta of the outgoing antineutrinos, $k_{\perp}$. This confirms that angular momentum conservation does not imply, as claimed in Ref. [9], that the momentum of the antineutrino has to be necessarily parallel to the magnetic field. In fact, it turns out that - as showed in Ref. [10] for large magnetic fields - the ratio $k_{\perp} / \sqrt{k_{\perp}^{2}+k_{z}^{2}}$ for outgoing antineutrinos tends to be relatively large. As expected, the derived expression for the integrated $\pi^{-} \rightarrow l \bar{\nu}_{l}$ width coincides exactly with the one found using the Landau gauge in Ref. [22]. This provides an explicit test of the gauge independence of this result.
It is worth noticing that Eq. (61) implies that for finite magnetic fields the decay width does not vanish in the limit $m_{l} \rightarrow 0$, i.e., it does not exhibit the helicity suppression found in the case of no external field. As shown in the present work, for a sufficiently large magnetic field (so that the outgoing charged lepton has to be in the LLL, and its mass can be neglected), helicity conservation only implies that the projection of the antineutrino momentum in the direction of the magnetic field should be antiparallel to the magnetic field. As a consequence, for large values of $B$ the ratio $\Gamma_{e} / \Gamma_{\mu}$ might change dramatically with respect to the $B=0$ value [10]. This could be interesting e.g., regarding the expected flavor composition of neutrino fluxes coming from the cores of magnetars and other stellar objects. In addition, for large $B$ the angular distribution of outgoing antineutrinos is expected to be highly anisotropic, showing a significant suppression in the direction of the external field.

\section{ACKNOWLEDGMENTS}

This work has been supported in part by Consejo Nacional de Investigaciones Científicas y Técnicas and Agencia Nacional de Promoción Científica y Tecnológica (Argentina), under Grants No. PIP17-700 and No. PICT1703-0571, respectively; by the National University of La Plata (Argentina), Project No. X824; by the Ministerio de Economía y Competitividad (Spain), under Contract No. FPA2016-77177-C2-1-P; and by the Centro de Excelencia Severo Ochoa Programme, Grant No. SEV2014-0398.

\section{APPENDIX A: ALTERNATIVE REPRESENTATION OF MAGNETIZED FERMION SPINORS IN THE SYMMETRIC GAUGE}

For completeness, in this Appendix we present an alternative way to express the charged fermion spinors. This form follows quite closely the Ritus representation notation often used in the Landau gauge (see e.g., Appendix A.3 of Ref. [22]). In fact, the spinors in Eq. (8) can also be written as

$$
\begin{aligned}
U_{l}^{s}(x, \breve{q}, \tau) & =\mathbb{N}_{\bar{q}}^{s}(x) u_{l}^{s}(\breve{q}, \tau), \\
V_{l}^{-s}(x, \breve{q}, \tau) & =\tilde{\mathbb{N}}_{\bar{q}}^{-s}(x) v_{l}^{-s}(\breve{q}, \tau),
\end{aligned}
$$

where $\bar{q}=\left(q^{0}, \breve{q}\right)$, with $q^{0}=E_{l}$. The spinors $u_{l}^{s}$ and $v_{l}^{-s}$ are given in the Weyl basis by

$$
u_{l}^{s}(\breve{q}, \tau)=\frac{1}{\sqrt{2\left(E_{l}+m_{l}\right)}}\left(\begin{array}{c}
\left(E_{l}+m_{l}+s \sqrt{2 n B_{e}} \tau_{2}-q_{z} \tau_{3}\right) \phi^{(\tau)} \\
\left(E_{l}+m_{l}-s \sqrt{2 n B_{e}} \tau_{2}+q_{z} \tau_{3}\right) \phi^{(\tau)}
\end{array}\right)
$$




$$
v_{l}^{-s}(\breve{q}, \tau)=\frac{1}{\sqrt{2\left(E_{l}+m_{l}\right)}}\left(\begin{array}{c}
\left(E_{l}+m_{l}-s \sqrt{2 n B_{e}} \tau_{2}-q_{z} \tau_{3}\right) \tilde{\phi}^{(\tau)} \\
-\left(E_{l}+m_{l}+s \sqrt{2 n B_{e}} \tau_{2}+q_{z} \tau_{3}\right) \tilde{\phi}^{(\tau)}
\end{array}\right)
$$

where $\tau_{i}$ are the Pauli matrices while $\phi^{(1) \dagger}=-\tilde{\phi}^{(2) \dagger}=$ $(1,0)$ and $\phi^{(2) \dagger}=\tilde{\phi}^{(1) \dagger}=(0,1)$. They satisfy the relations

$$
\begin{aligned}
& \sum_{\tau=1,2} u_{l}^{s}(\breve{q}, \tau) \bar{u}_{l}^{s}(\breve{q}, \tau)=\ddot{q}_{s}+m_{l}, \\
& \sum_{\tau=1,2} v_{l}^{-s}(\breve{q}, \tau) \bar{v}_{l}^{-s}(\breve{q}, \tau)=\not_{-s}-m_{l},
\end{aligned}
$$

where $\hat{q}_{s}^{\mu}=\left(E_{l}, 0,-s \sqrt{2 n B_{e}}, q_{z}\right)$. In Eq. (A1), $\mathbb{N}_{\bar{q}}^{s}(x)$ and $\tilde{\mathbb{N}}_{\bar{q}}^{-s}(x)$ are the symmetric gauge equivalents of the Landau gauge Ritus functions. They are solutions of the eigenvalue equation

$$
\not D^{2} \mathbb{N}_{\bar{q}}^{s}(x)=-\left[E_{l}^{2}-2 n B_{e}-q_{z}^{2}\right] \mathbb{N}_{\bar{q}}^{s}(x)
$$

where $\not D=\not \partial-i s B_{e}\left(r_{x} \gamma^{2}-r_{y} \gamma^{1}\right)$. The explicit form of these functions is

$$
\mathbb{N}_{\bar{q}}^{s}(x)=\sum_{\lambda= \pm} N_{\bar{q}, \lambda}^{s}(x) \Delta^{\lambda}, \quad \tilde{\mathbb{N}}_{\bar{q}}^{-s}(x)=\sum_{\lambda= \pm} N_{\bar{q},-\lambda}^{-s}(x)^{*} \Delta^{\lambda}
$$

where $\Delta^{ \pm}=\left(1 \pm i \gamma^{1} \gamma^{2}\right) / 2$ and

$$
N_{\bar{q}, \lambda}^{s}(x)=W_{\left(E_{l}, n_{s \lambda}, v, q_{z}\right)}^{s}(x),
$$

$W_{\bar{q}}^{s}(x)$ being given by Eq. (3). Here the non-negative integer index $n_{s \lambda}$ is related to the quantum number $n$ by $n_{s \pm}=n-(1 \mp s) / 2$.

\section{APPENDIX B: NEUTRINO FIELD IN A CYLINDRICAL BASIS}

It is usual to write the neutrino field as a linear combination of operators of well-defined linear momentum $\vec{k}$. However, this is not very convenient for the purpose of the present work. As mentioned in the main text, we are interested in dealing with the decay of charged pions in the presence of an external field using the symmetric gauge. Thus, as in the case of charged pions and leptons, it is more convenient to introduce an expansion of the neutrino field using cylindrical coordinates $\rho, \phi$ and $z$, where $z$ is a spacial axis parallel to the magnetic field. We can then expand the usual plane wave functions in terms of eigenfunctions of the $z$ component of the orbital momentum, $l_{z}$. Next, we can couple these wave functions to the eigenstates of $S_{z}$ ( $z$ component of the spin operator), and write the neutrino field in terms of operators with "good" total angular momentum $j_{z}=l_{z}+S_{z}$. The resulting expansion reads

$$
\begin{aligned}
\psi_{\nu_{l}}(x)= & \sum_{J=-\infty}^{\infty} \int \frac{d k_{z} d k_{\perp} k_{\perp}}{(2 \pi)^{2} 2 E_{\nu_{l}}}\left[b(\breve{k}, L) U_{\nu_{l}}(x, \breve{k}, L)\right. \\
& \left.+d(\breve{k}, R)^{\dagger} V_{\nu_{l}}(x, \breve{k}, R)\right]
\end{aligned}
$$

where $\breve{k}=\left(J, k_{\perp}, k_{z}\right)$ and $E_{\nu_{l}}=E_{\bar{\nu}_{l}}=\sqrt{k_{\perp}^{2}+k_{z}^{2}}$. Here, $J$ is an integer number, while $k_{\perp}$ and $k_{z}$ are real numbers, with $k_{\perp}>0$. In the Weyl basis, the spinors $U_{\nu_{l}}$ and $V_{\nu_{l}}$ are given by

$\begin{aligned} U_{\nu_{l}}(x, \breve{k}, L)= & -i^{J} e^{-i\left(E_{\bar{\nu}_{l}} t-k_{z} r_{z}\right)} \\ & \times e^{-i J \phi}\left(\begin{array}{c}\sqrt{E_{\bar{\nu}_{l}}-k_{z}} J_{J}\left(k_{\perp} \rho\right) \\ i \sqrt{E_{\bar{\nu}_{l}}+k_{z}} e^{i \phi} J_{J-1}\left(k_{\perp} \rho\right) \\ 0 \\ 0\end{array}\right),\end{aligned}$

$$
\begin{aligned}
V_{\nu_{l}}(x, \breve{k}, R)= & -(-i)^{J} e^{i\left(E_{\bar{\nu}_{l}} t-k_{z} r_{z}\right)} \\
& \times e^{-i \jmath \phi}\left(\begin{array}{c}
\sqrt{E_{\bar{\nu}_{l}}-k_{z}} J_{J}\left(k_{\perp} \rho\right) \\
-i \sqrt{E_{\bar{\nu}_{l}}+k_{z}} e^{i \phi} J_{J-1}\left(k_{\perp} \rho\right) \\
0 \\
0
\end{array}\right) .
\end{aligned}
$$

Note that, as it is clear from the explicit form of the spinors, in the expansion we have already taken into account that neutrinos (antineutrinos) are lefthanded (righthanded). The creation and annihilation operators satisfy

$$
\begin{aligned}
& \left\{b(\breve{k}, L), b\left(\breve{k}^{\prime}, L\right)^{\dagger}\right\}=\left\{d(\breve{k}, R), d\left(\breve{k}^{\prime}, R\right)^{\dagger}\right\}=2 E_{\bar{\nu}_{l}}(2 \pi)^{2} \delta_{\breve{k}, \breve{k}^{\prime}}, \\
& \left\{b(\breve{k}, L), d\left(\breve{k}^{\prime}, R\right)^{\dagger}\right\}=\left\{d(\breve{k}, L)^{\dagger}, b\left(\breve{k}^{\prime}, R\right)^{\dagger}\right\}=0,
\end{aligned}
$$

where

$$
\delta_{\breve{k}, \breve{k}^{\prime}}=\delta_{\jmath J^{\prime}} \frac{\delta\left(k_{\perp}-k_{\perp}^{\prime}\right)}{k_{\perp}} \delta\left(k_{z}-k_{z}^{\prime}\right)
$$

It can be seen that the spinors in Eqs. (B2) and (B3) satisfy the orthogonality relations 


$$
\begin{aligned}
\int d^{3} r U(x, \breve{k}, L)^{\dagger} U\left(x, \breve{k}^{\prime}, L\right) & =\int d^{3} r V(x, \breve{k}, R)^{\dagger} V\left(x, \breve{k}^{\prime}, R\right) \\
& =2 E_{\bar{\iota}_{l}}(2 \pi)^{2} \widetilde{\delta}_{\breve{k}, \breve{k}^{\prime}} \\
\int d^{3} r U(x, \breve{k}, L)^{\dagger} V\left(x, \breve{k}^{\prime}, R\right) & =\int d^{3} r V(x, \breve{k}, R)^{\dagger} U\left(x, \breve{k}^{\prime}, L\right) \\
& =0 .
\end{aligned}
$$

Using methods similar to those mentioned in Sec. II C it can be shown that, given a set of quantum numbers $\left(J, k_{\perp}, k_{z}\right)$, the eigenvalue of the total angular momentum operator $\tilde{j}_{z}$ acting on a neutrino state is $j_{z}^{\left(\nu_{l}\right)}=-(j-1 / 2)$, while for an antineutrino state one has $j_{z}^{\left(\bar{\nu}_{l}\right)}=j-1 / 2$.

\section{APPENDIX C: THE RADIAL INTEGRAL}

In this Appendix we quote the result for the radial integral appearing in Eq. (43). It can be calculated using the relation (see Eq. (5) of Ref. [25])

$$
\begin{gathered}
\int d x x^{\nu+1} e^{-\alpha x^{2}} L_{m}^{\nu-\sigma}\left(\alpha x^{2}\right) L_{n}^{\sigma}\left(\alpha x^{2}\right) J_{\nu}(x y) \\
=\frac{(-1)^{m+n}}{2 \alpha}\left(\frac{y}{2 \alpha}\right)^{\nu} \exp \left(-\frac{y^{2}}{4 \alpha}\right) \\
\quad \times L_{m}^{\sigma-m+n}\left(\frac{y^{2}}{4 \alpha}\right) L_{n}^{\nu-\sigma+m-n}\left(\frac{y^{2}}{4 \alpha}\right) \\
\quad \times[y>0, \operatorname{Re} \alpha>0, \operatorname{Re} \nu>-1],
\end{gathered}
$$

together with (see Eq. (3.6.2) of Ref. [26])

$$
L_{v}^{n-v}(x)=\frac{n !}{v !}(-x)^{v-n} L_{n}^{v-n}(x)
$$

which is valid for all the values of $n$ and $v$ we are interested in. We get

$$
\begin{aligned}
\mathcal{I}(\ell, l, n, v)= & 2 \pi(-1)^{l+v} \sqrt{\frac{l ! n !}{\ell ! v !}}\left(\frac{k_{\perp}^{2}}{2 B_{e}}\right)^{\frac{(\ell-l)-(n-v)}{2}} \\
& \times e^{-\frac{k_{\perp}^{2}}{2 B_{e}}} L_{l}^{v-l}\left(\frac{k_{\perp}^{2}}{2 B_{e}}\right) L_{n}^{\ell-n}\left(\frac{k_{\perp}^{2}}{2 B_{e}}\right) .
\end{aligned}
$$

\section{APPENDIX D: SUM OVER THE QUANTUM NUMBER $v$ OF THE OUTGOING CHARGED LEPTONS}

In this Appendix we will prove the validity of Eq. (60). From the definition of $F_{l, v}(x)$ in Eq. (45) we have

$$
\sum_{v=0}^{\infty} F_{l, v}(x)^{2}=e^{-2 x} S_{l}(x),
$$

where

$$
S_{l}(x)=\sum_{v=0}^{\infty} \frac{\imath !}{v !} x^{v-l} L_{l}^{v-l}(x)^{2} .
$$

We want to show that $S_{l}(x)=e^{x}$, for all $l \epsilon \mathbf{N}$. For $\imath=0$ one has $L_{0}^{v}(x)=1$, therefore the relation is clearly satisfied. For $\imath=1$, using $L_{1}^{v-1}(x)=v-x$, one has

$$
\begin{aligned}
S_{1}(x) & =x+\sum_{v=1}^{\infty} \frac{x^{v-1}}{v !}(v-x)^{2} \\
& =x+\sum_{v=1}^{\infty}\left[\frac{x^{v+1}}{v !}+\frac{v x^{v-1}}{(v-1) !}-\frac{2 x^{v}}{(v-1) !}\right],
\end{aligned}
$$

from which $S_{1}(x)=e^{x}$ easily follows. For $\imath \geq 2$, let us calculate the derivative of $S_{l}(x)$ with respect to $x$. Using Eq. (C2) one can write

$S_{l}(x)=\sum_{v=0}^{l-1} \frac{v !}{\imath !} x^{l-v} L_{v}^{l-v}(x)^{2}+\sum_{v=l}^{\infty} \frac{\imath !}{v !} x^{v-l} L_{l}^{v-l}(x)^{2}$.

In this way, using the general relation

$$
\frac{d L_{n}^{\alpha}(x)}{d x}=-L_{n-1}^{\alpha+1}(x),
$$

one has

$\frac{d S_{l}(x)}{d x}=\frac{x^{l-1}}{(l-1) !}+S_{l}^{(1)}(x)-2 L_{l}(x) L_{l-1}^{1}(x)+S_{l}^{(2)}(x)$,

where

$$
\begin{aligned}
S_{l}^{(1)}(x)= & \sum_{v=1}^{l-1} \frac{v !}{\imath !}\left[(l-v) x^{l-v-1} L_{v}^{l-v}(x)^{2}\right. \\
& \left.-2 x^{l-v} L_{v}^{l-v}(x) L_{v-1}^{l-v+1}(x)\right], \\
S_{l}^{(2)}(x)= & \sum_{v=l+1}^{\infty} \frac{\imath !}{v !}\left[(v-\imath) x^{v-l-1} L_{l}^{v-l}(x)^{2}\right. \\
& \left.-2 x^{v-l} L_{l}^{v-l}(x) L_{l-1}^{v-l+1}(x)\right] .
\end{aligned}
$$

The sums in Eqs. (D7) can be worked out using the relations

$$
\alpha L_{n}^{\alpha}(x)=x L_{n}^{\alpha+1}(x)+(n+1) L_{n+1}^{\alpha-1}(x)
$$

and

$$
L_{n+1}^{\alpha}(x)=L_{n+1}^{\alpha+1}(x)-L_{n}^{\alpha+1}(x) .
$$

For the first sum one has 


$$
\begin{aligned}
S_{l}^{(1)}(x) & =\sum_{v=1}^{l-1}\left[(l-v) L_{v}^{l-v}(x)-2 x L_{v-1}^{l-v+1}(x)\right] \frac{v !}{\imath !} x^{l-v-1} L_{v}^{l-v}(x) \\
& =\sum_{v=1}^{l-1}\left[\frac{v !}{\imath !} x^{l-v} L_{v}^{l-v}(x)^{2}+\frac{(v+1) !}{\imath !} x^{l-v-1} L_{v+1}^{l-v-1}(x) L_{v}^{l-v}(x)-\frac{v !}{\imath !} x^{l-v} L_{v}^{l-v}(x) L_{v-1}^{l-v+1}(x)\right] \\
& =L_{l}(x) L_{l-1}^{1}(x)-(l-x) \frac{x^{l-1}}{\imath !}+\sum_{v=1}^{l-1} \frac{v !}{\imath !} x^{l-v} L_{v}^{l-v}(x)^{2}
\end{aligned}
$$

where we have used $L_{0}^{l}(x)=1, L_{1}^{l-1}(x)=\imath-x$. The second sum is given by

$$
\begin{aligned}
S_{l}^{(2)}(x) & =\sum_{v=l+1}^{\infty}\left[\frac{\imath !}{v !} x^{v-l} L_{l}^{v-l}(x)^{2}+\frac{(l+1) !}{v !} x^{v-l-1} L_{l+1}^{v-l-1}(x) L_{l}^{v-l}(x)-\frac{\imath !}{v !} x^{v-l} L_{l}^{v-l}(x) L_{l-1}^{v-l+1}(x)\right] \\
& =\sum_{v=l+1}^{\infty} \frac{\imath !}{v !} x^{v-l} L_{l}^{v-l}(x)^{2}+L_{l+1}(x) L_{l}^{1}(x)+\sum_{v=l+2}^{\infty} \frac{\imath !}{v !} x^{v-l-1}\left[(l+1) L_{l+1}^{v-l-1}(x) L_{l}^{v-l}(x)-v L_{l-1}^{v-l}(x) L_{l}^{v-l-1}(x)\right] .
\end{aligned}
$$

Here one can use the relation

$$
(n+1) L_{n+1}^{\alpha}(x)=(n+\alpha+1) L_{n}^{\alpha}(x)-x L_{n}^{\alpha+1}(x)
$$

together with Eq. (D9) to obtain

$$
(l+1) L_{l+1}^{v-l-1}(x) L_{l}^{v-l}(x)-v L_{l-1}^{v-l}(x) L_{l}^{v-l-1}(x)=v L_{l}^{v-l-1}(x)^{2}-x L_{l}^{v-l}(x)^{2} .
$$

Replacing into Eq. (D13) and performing an adequate change in the index of the sum it is easy to arrive at

$$
S_{l}^{(2)}(x)=\sum_{v=l+1}^{\infty} \frac{\imath !}{v !} x^{v-l} L_{l}^{v-l}(x)^{2}+L_{l+1}(x) L_{l}^{1}(x)+\frac{x}{l+1} L_{l}^{1}(x)^{2} .
$$

Now replacing the expressions for $S_{l}^{(1)}(x)$ and $S_{l}^{(2)}(x)$ in Eqs. (D12) and (D13) into Eq. (D6), and using Eq. (D9), one obtains

$$
\frac{d S_{l}(x)}{d x}=\sum_{v=0}^{l-1} \frac{v !}{\imath !} x^{l-v} L_{v}^{l-v}(x)^{2}+\sum_{v=l}^{\infty} \frac{\imath !}{v !} x^{v-l} L_{l}^{v-l}(x)^{2}+L_{l}^{1}(x)\left[L_{l+1}(x)-L_{l}(x)+\frac{x}{\imath+1} L_{l}^{1}(x)\right],
$$

where the last term on the right-hand side vanishes, according to Eq. (D14). Thus, taking into account the definition of $S_{l}(x)$ in Eq. (D4), it is seen that $d S_{l}(x) / d x=S_{l}(x)$. Since, in addition, for all $\iota$ one has $S_{l}(0)=L_{l}(0)^{2}=1$, one obtains $S_{l}(x)=e^{x}$. From Eq. (D1) one has finally

$$
\sum_{v=0}^{\infty} F_{l, v}(x)^{2}=e^{-x}
$$

[1] D. E. Kharzeev, K. Landsteiner, A. Schmitt, and H. U. Yee, Lect. Notes Phys. 871, 1 (2013).

[2] J. O. Andersen, W. R. Naylor, and A. Tranberg, Rev. Mod. Phys. 88, 025001 (2016).
[3] V. A. Miransky and I. A. Shovkovy, Phys. Rep. 576, 1 (2015).

[4] D. Grasso and H. R. Rubinstein, Phys. Rep. 348, 163 (2001). 
[5] D. E. Kharzeev, L. D. McLerran, and H. J. Warringa, Nucl. Phys. A803, 227 (2008); V. Skokov, A. Y. Illarionov, and V. Toneev, Int. J. Mod. Phys. A 24, 5925 (2009); V. Voronyuk, V. Toneev, W. Cassing, E. Bratkovskaya, V. Konchakovski, and S. Voloshin, Phys. Rev. C 83, 054911 (2011).

[6] R. C. Duncan and C. Thompson, Astrophys. J. 392, L9 (1992); C. Kouveliotou et al., Nature (London) 393, 235 (1998).

[7] V. P. Gusynin, V. A. Miransky, and I. A. Shovkovy, Phys. Rev. Lett. 73, 3499 (1994); 76, 1005(E) (1996).

[8] G. S. Bali, F. Bruckmann, G. Endrődi, Z. Fodor, S. D. Katz, S. Krieg, A. Schafer, and K. K. Szabo, J. High Energy Phys. 02 (2012) 044; G. S. Bali, F. Bruckmann, G. Endrődi, Z. Fodor, S. D. Katz, and A. Schafer, Phys. Rev. D 86, 071502 (2012).

[9] G. S. Bali, B. B. Brandt, G. Endrődi, and B. Gläßle, Phys. Rev. Lett. 121, 072001 (2018).

[10] M. Coppola, D. Gomez Dumm, S. Noguera, and N. N. Scoccola, arXiv:1908.10765.

[11] E. Waxman and J. N. Bahcall, Phys. Rev. Lett. 78, 2292 (1997).

[12] B. Zhang, Z. G. Dai, and P. Meszaros, Astrophys. J. 595, 346 (2003).

[13] A. I. Nikishov and V. I. Ritus, Zh. Eksp. Teor. Fiz. 46, 776 (1964) [Sov. Phys. JETP 19, 529 (1964)], http://www.jetp .ac.ru/cgi-bin/e/index/e/19/2/p529?a=list.
[14] A. I. Nikishov and V. I. Ritus, Zh. Eksp. Teor. Fiz. 46, 1768 (1964) [Sov. Phys. JETP 19, 1191 (1964)], http://www.jetp .ac.ru/cgi-bin/e/index/e/19/5/p1191?a=list.

[15] J. J. Matese and R. F. O'Connell, Phys. Rev. 180, 1289 (1969).

[16] L. Fassio-Canuto, Phys. Rev. 187, 2141 (1969).

[17] J. O. Andersen, J. High Energy Phys. 10 (2012) 005.

[18] Y. A. Simonov, Yad. Fiz. 79, 277 (2016) [Phys. At. Nucl. 79, 455 (2016)]

[19] H. Liu, X. Wang, L. Yu, and M. Huang, Phys. Rev. D 97, 076008 (2018).

[20] M. Coppola, D. Gomez Dumm, and N. N. Scoccola, Phys. Lett. B 782, 155 (2018).

[21] M. Coppola, D. Gomez Dumm, S. Noguera, and N. N. Scoccola, Phys. Rev. D 100, 054014 (2019).

[22] M. Coppola, D. Gomez Dumm, S. Noguera, and N. N. Scoccola, Phys. Rev. D 99, 054031 (2019).

[23] M. Wakamatsu, Y. Kitadono, and P.-M. Zhang, Ann. Phys. (N.Y.) 392, 287 (2018).

[24] A. A. Sokolov, I. M. Ternov, and C. W. Kilmister, Radiation from Relativistic Electrons (AIP, New York, 1986).

[25] K. S. Kolbig and H. Scherb, J. Comput. Appl. Math. 71, 357 (1996).

[26] B. Doman, The Classical Orthogonal Polynomials (World Scientific, Singapore, 2015). 